\title{
Review of: "Impact of type of reconstructed residence on social participation and mental health of population displaced by disasters"
}

\author{
Shinichi Egawa \\ 1 Tohoku University
}

Potential competing interests: I collaborate with the authors in several projects in disaster medicine and public health research. Thus, my review on this article is a favor from closely working collaborator.

Disaster puts significant mental health load to the affected people. Sendai Framework for Disaster Risk Reduction 2015-2030 is the first international framework that disaster affects the health physically and mentally, although this fact was recognized from thousands of years ago. This paper “Impact of type of reconstructed residence on social participation and mental health of population displaced by disasters (Suzuki T, et al 2021, Scientific Reports)" have discovered that the psychosocial indicators are worse in the people living in "disaster-recovery public condominiums" compared to the people living in other types of relocation using the cohort study data of Shichigahama Town. Cohort study allows us to elucidate the longterm effect of disasters not only by following longer period, but by the active participation of the residents with written informed consent. Thus, by the careful cross-sectional analysis, we can find valuable evidence to improve the resilience of society.

People will not select their place of relocation randomly. As the authors state, the ratio of sex and age did not differ between the four types of reconstructed residences, but the number of family members per household was significantly lower in people who moved into disaster-recovery public condominiums, suggesting that the possibility of social isolation could exist when they decided to relocate into the disaster recovery public condominiums.

The sleep disturbance was not different by the type of residence. The authors state that lower levels of social interaction and elevated rate of depressive state in people who live in the public condominiums regardless of the current employment status or the age group, but were especially remarkable among the younger adult disaster-affected population aged < 65 years. The social roles and activities in the age group could be analyzed further. Even the age group had threshold at age of 65, the social role, activities and future perspectives will be different between the families with age 50 s and age 30 s. There might be some effects of number of households. If the family had grandparents, or small children, how will they choose the type of relocation. The role of family members could be the first level social capitol to encourage to choose "living in the same place", "relocate to a higher place with the community", or "reconstruct a new house at remote area" although it may take additional time. That will give a hint when the local and national government think the "effective intervention" recommended by the authors. 
Forming a family or comfortable human relationship with a friend which was lost by the disaster in a moment, will take time but seem to be a good solution for the affected people.

About the terminology, I recommend to use "disaster affected people" rather than "disaster victims", and "evacuation centers" rather than "refugee camps" in order to describe the situation correctly. United Nations Office for Disaster Risk Reduction (UNDRR) is indicating the terminology (https://www.undrr.org/terminology) so that the all sectors can communicate better. Health sector is an essential sector in the disaster risk reduction. The International Conference on the Implementation of the Health Aspects of the Sendai Framework for Disaster Risk Reduction 2015-2030 recommended seven measures (Bangkok Principles https://www.preventionweb.net/publication/bangkok-principlesimplementation-health-aspects-sendai-framework-disaster-risk) to enhance the cooperation and harmonization between health sector and other sectors in disaster risk reduction.

WHO declared the Health Emergency and Disaster Risk Management (H-EDRM) Framework (https://www.who.int/hac/techguidance/preparedness/health-emergency-and-disaster-risk-managementframework-eng.pdf) to improve the resilience of society and published the Guidance on Research Methods for H-EDRM (https://extranet.who.int/kobe_centre/en/projectdetails/GUIDANCE_ResearchMethods_HealthEDRM) in 2021. The initiative and commitment of international community encourage us to utilize the existing knowledge and future research including cohort studies to improve the science and implementation of resilience. 